Supplement of Atmos. Chem. Phys., 14, 4441-4457, 2014

http://www.atmos-chem-phys.net/acp-14-4441-2014/

doi:10.5194/acp-14-4441-2014-supplement

(C) Author(s) 2014. CC Attribution 3.0 License.

(c) (1)

Supplement of

\title{
Atmospheric deposition of polybromodiphenyl ethers in remote mountain regions of Europe
}

L. Arellano et al.

Correspondence to: P. Fernández (pilar.fernandez@idaea.csic.es) 
Table S1. IUPAC name and Chemical Abstract Service (CAS) number of target PBDEs (see Figure S1 for chemical structure).

\begin{tabular}{|c|c|c|c|}
\hline & & IUPAC name & CAS N $^{\circ}$ \\
\hline \multirow[t]{2}{*}{ Tribromo BDEs } & BDE17 & 2,2',4-Tribromodiphenyl ether & $147217-75-2$ \\
\hline & BDE28 & 2,4,4'-Tribromodiphenyl ether & $41318-75-6$ \\
\hline \multirow[t]{3}{*}{ Tetrabromo BDEs } & BDE47 & $2,2^{\prime}, 4,4^{\prime}$-Tetrabromodiphenyl ether & $5436-43-1$ \\
\hline & BDE66 & $2,3^{\prime}, 4,4^{\prime}$-Tetrabromodiphenyl ether & $189084-61-5$ \\
\hline & BDE71 & $2,3^{\prime}, 4^{\prime}, 6$-Tetrabromodiphenyl ether & $189084-62-6$ \\
\hline \multirow[t]{3}{*}{ Pentabromo BDEs } & BDE85 & $2,2^{\prime}, 3,4,4^{\prime}$-Pentabromodiphenyl ether & $182346-21-0$ \\
\hline & BDE99 & $2,2^{\prime}, 4,4^{\prime}, 5$-Pentabromodiphenyl ether & $60328-60-9$ \\
\hline & BDE100 & $2,2^{\prime}, 4,4^{\prime}, 6$-Pentabromodiphenyl ether & $189084-64-8$ \\
\hline \multirow[t]{3}{*}{ Hexabromo BDEs } & BDE153 & $2,2^{\prime}, 4,4^{\prime}, 5,5^{\prime}$-Hexabromodiphenyl ether & $68631-49-2$ \\
\hline & BDE154 & $2,2^{\prime}, 4,4^{\prime}, 5,6^{\prime}$-Hexabromodiphenyl ether & $207122-15-4$ \\
\hline & BDE138 & $2,2^{\prime}, 3,4,4^{\prime}, 5^{\prime}$-Hexabromodiphenyl ether & $182677-30-1$ \\
\hline \multirow[t]{2}{*}{ Heptabromo BDEs } & BDE183 & $2,2^{\prime}, 3,4,4^{\prime}, 5^{\prime}, 6$-Heptabromodiphenyl ether & $207122-16-5$ \\
\hline & BDE190 & 2,3,3',4,4',5,6-Heptabromodiphenyl ether & 30338 \\
\hline Decabromo BDE & BDE209 & Decabromodiphenyl ether & $1163-19-5$ \\
\hline
\end{tabular}


Table S2. Method detection (MDL) and quantification (MQL) limits in $\mathrm{ng} \mathrm{m}^{-2}$

\begin{tabular}{lcc}
\hline & MDL & MQL \\
\hline BDE28 & 1.14 & 1.15 \\
BDE71 & 0.78 & 0.92 \\
BDE47 & 3.12 & 3.89 \\
BDE66 & 1.57 & 1.91 \\
BDE100 & 1.69 & 2.26 \\
BDE99 & 3.18 & 3.39 \\
BDE154 & 0.66 & 0.85 \\
BDE153 & 0.94 & 1.03 \\
BDE183 & 1.41 & 1.44 \\
BDE209 & 40.9 & 42.0 \\
\hline
\end{tabular}

Table S3. BDE congeners in blanks (average of all method and field blanks) in $\mathrm{ng} \mathrm{m}^{-2}$

\begin{tabular}{ll}
\hline BDE28 & 1.13 \\
BDE71 & 0.56 \\
BDE47 & 1.97 \\
BDE66 & 1.06 \\
BDE100 & 0.83 \\
BDE99 & 2.88 \\
BDE154 & 0.33 \\
BDE153 & 0.81 \\
BDE183 & 1.36 \\
BDE209 & 39.2 \\
\hline
\end{tabular}


Figure S1. General chemical structure of PBDEs $(m+n=1-10)$<smiles>Brc1ccc(Oc2ccccc2)cc1</smiles> 
Figure S2. Correlation plots between individual BDE congeners $\left(\mathrm{ng} \mathrm{m}^{-2} \mathrm{mo}^{-1}\right)$, particle fluxes $\left(\mathrm{mg} \mathrm{m}^{-2} \mathrm{mo}^{-1}\right)$, temperature $\left({ }^{\circ} \mathrm{C}\right)$ and precipitation $(\mathrm{mm})$ in Skalnate. Log-transformed data excepting for meteorological variables.

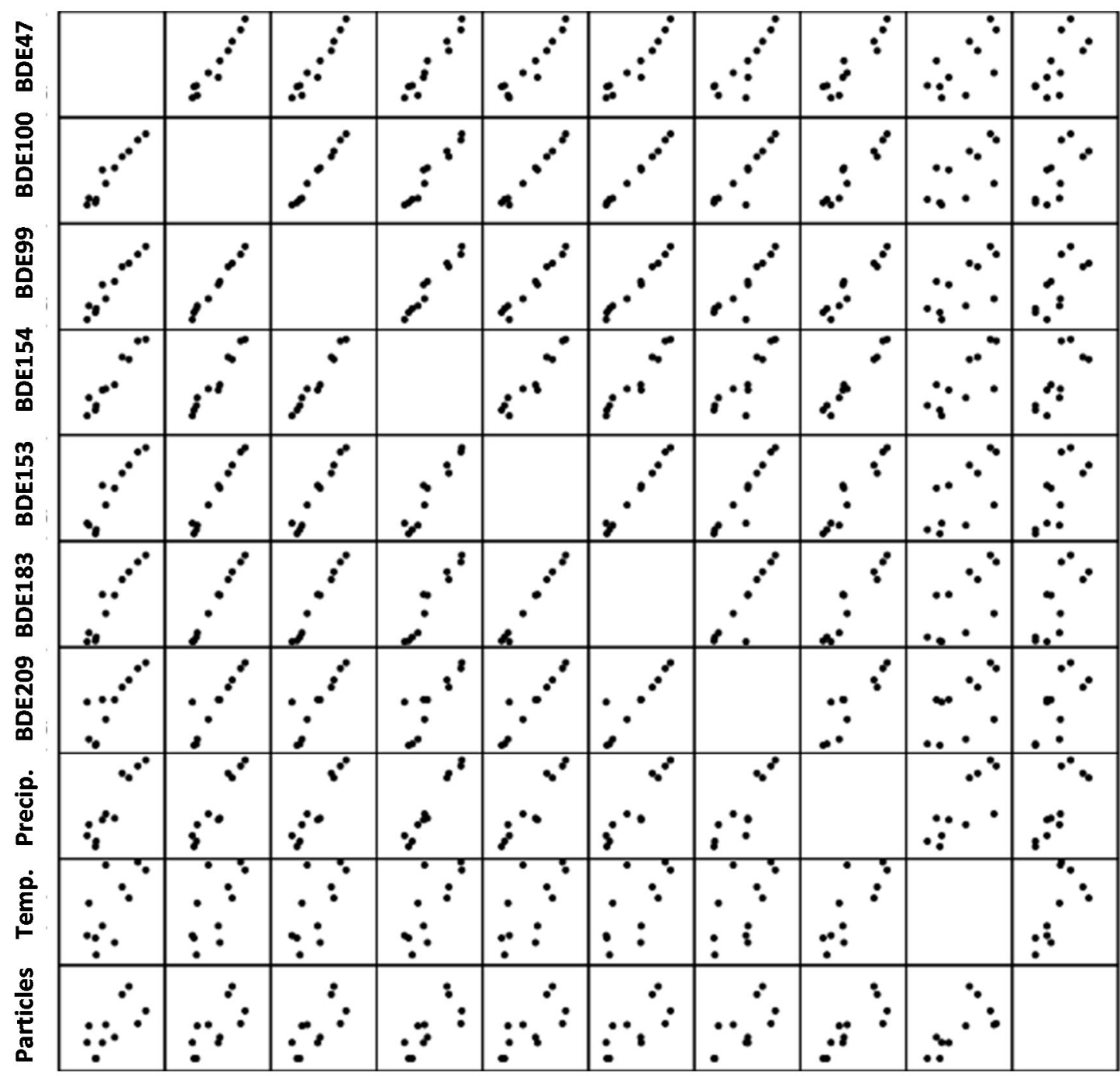

BDE47 BDE100 BDE99 BDE154 BDE153 BDE183 BDE209 Precip. Temp. Particles 